\title{
DISCLAIMER
}

This report was prepared as an eccount of work sponsorod by an egency of the United States Government. Neither the United Sintes Government nor any asency thereof, nor any of their employeet mates any warranty, express or implied, or assumes anj legal liability or responzibility for the accuracy, completeness, or usefulness of any information, apparatus, product, or process disclosed, of represents that its use would not infringe privately owned sights. Reference herein to any spexific commercial product, provill, or semice by trade name, trademark, manufacturer, or otherwise does not necessurily constjtute or imply its endorsement. reconmexdation, or favoring by the United States Governonest or eny atency therbof. The views and opinions of authors expressed herein do not necessarily siate or reflect those of the United States Government or any agency thereot.

SLAC! AP -98

January 1995

(AP)

\section{Study of the Trapped Modes at the Vacuum Ports*}

\author{
S. Heifets and G. Stupakov \\ Stanford Linear Accelerator Center, Stanford University, Stanford, CA 94309
}

Pumping ports in PEI'][ vacuun chanluer will be slipelded with slots cut in the walls of the beam pipe. The number of slots $n_{s}$, their length $l$, and width $w$ are defined by the required pumping conduclance. The prescot design has 40 slots around the stainless stecl round pipe in the straight sections, and, for the rectangulaf copper chamber of the ares, there are 18 slots plared in upper and lower decks.

Hele we want to acldress two polential problems related to the slots: radiation of the TE HOM-s through the slots, and generation of the trapped modes.

\section{Radiation through the slots}

Radiation through the slots is inducerl by the fied of the beim and by the IIOM propagating in the vacum chanber. 'I'lue excessive power radiated by the slots may be damaging to the pumps.

The encrgy radiated by a Giassian bunch with number of partickes $N_{B B}$, and rms length $\sigma_{D}$ through a longitudinal slot in a thitn wall of a beam pipe with radius $b$ is ${ }^{[1]}$

$$
\Delta U=\frac{N_{B^{\prime}}^{2} c^{2}\left(\alpha_{c}^{2}+\alpha_{m}^{2}\right)}{3 \pi(2 \pi b)^{2}} \int_{-\infty}^{\infty} d \frac{\omega}{c}\left(\frac{\omega}{c}\right)^{4}|\rho(\omega)|^{2}
$$

where $\alpha_{c, n}$ are polarizabilities of tlec slot, and $\rho(\omega)=\operatorname{cr} p\left[-\left(\omega^{\prime} \sigma_{B} / c\right)^{2}\right]$.

Radiation induced by the ficlal of a bunch of by TM JOML-s generated by a bunch somewhere upstieam from the slot is given mostly by the trausverse magnetic

* Work supported by Dopartmont of Energy coutrnet UE-AC03-76SF00515

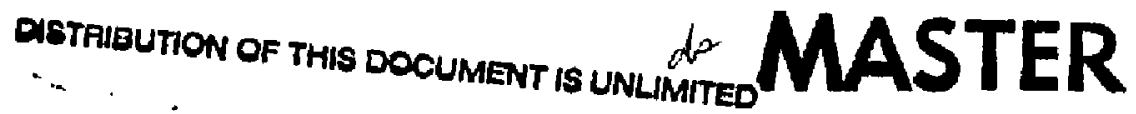


polarizability. For a long slot in a thick wall

$$
\alpha_{e}=\alpha_{m}=l w^{2} / 2 \pi
$$

The radiation is exponentially small provided the slot is narrow compared with the wall thickness $d, 2 \pi d / w>1$ :

$$
\Delta U=\frac{N_{\vec{B}}^{2} e^{2}}{2 \sqrt{\pi} \sigma_{B}(2 \pi b)^{2}}\left(\frac{l w^{2}}{2 \pi \sigma_{B}^{2}}\right)^{2} e^{-2 \pi d / u}
$$

For $2 d y<\pi \sigma^{2}$, the exponent is given by the attenuation in the slot rather than by the roll-off of the bunch spectrum.

However. the radiation from a slot induced by TE HOM-s is much larger because it is given by the longitudinnl polarizability proportional to $l^{3}$. The ratio of the radiated power to the power of the incident TE wave can be derived easily from Kurennoy's result ${ }^{\text {II! }}$

$$
\frac{\Delta P}{P_{i n}}=\frac{2}{3 \pi}\left(\frac{\omega}{c}\right)^{3} \frac{k_{c}^{2} a_{m}^{2}}{g a b}
$$

where $k_{c}=\omega_{c} / c$ is given by the cutoff frequency $\omega_{c}, q$ is propagating constant, and

$$
\alpha_{m}=\frac{\pi l^{3}}{24(\ln 4(/ w-1)}
$$

Note, that $110 \mathrm{O}$ ean not propagate in the beam pipe with finite conductivity at the cuttoff frequency. The worst case corresponds to digh freciucncies. At the maximum frequency of a bunch spectrum $\omega / c=1 / \sigma$, which is well alsove cutoff for short bunches, Eq. (4) can be simplified to

$$
\frac{\Delta P}{P_{i n}}=\frac{2 \pi}{3}\left(\frac{\pi}{24}\right)^{2} \frac{\sigma}{a \mathrm{In}^{2}(4 l / w)}\left(\frac{l^{2}}{\sigma_{D} b^{3}}\right)^{3} .
$$

$\mathrm{Eq} .(5)$ is written for a rectangular beam pipe with dimensions $a \times b, b^{2}<<a^{2}$. 
Fol the PEP-II arcs, $l=10 \mathrm{~cm}, a=10 \mathrm{~cm}, b=5 \mathrm{~cm}, t=0.3 \mathrm{~cm}$, and $\sigma_{B}=1$ $\mathrm{cm}$ the ratio is close to one. This means that a slot is transparent for a TE modeThe power in the TE HOMs is unknowil. Contrary to TM IIOM-s, they are not coupled directly to the bean and may be generated by mode conversion due to the scalerring of the TM modes in the bcam pipe, or by broad-band losses to the hybrid modes mostly in the RF cavities. A TM $m=0$ mode may mix with a TE mode only in a cavity without cylindrical symmetry. The TE component of the hybrid mode is proportjonal to the ratio of the surface areas of couplers, damping ports, etc to the total surface area of a cavity. We estimate this ratio as less than $S=5 \%$. A loss factor of TM modes above cutof feequency of a RF cavities is $0.12 \mathrm{~V} / \mathrm{pC}$. Hence, 24 RF cavities of the HER cau generate TE modes with power

$$
P=Z_{0} I_{B} \frac{h_{1} s_{b}}{4 \pi} S=4.5 \mathrm{~kW}
$$

at the beam current $l_{b}=3 \mathrm{~A}$.

The dipole modes in the RF cavities may be fully compled with TE nodes, but they are not exited by a beam with a zero offset " 6 . Therefore, the loss to this modes is of the sirder of $r_{b} / 6$ compared to the lossces to the $m=0$ modes.

The power $\mathrm{Eq}_{\text {q. }}$ (6) will go through the slots of few closest to the RF cavities pumping ports and may damage them. That can be avoided by covering slots with a mesh of holes with small diameter from the outer side of the slots as it will be done for the dipole screens.

\section{Mode trapping}

Another issue is mode trapping. The leak of the magnetic ficld into a slot may shift the frequency of a node cluse to the cuttofl frectucney $\omega_{c}$ below the cutoff producing a trapped mode which exponent inlly decays at the large distances from the slot,

$$
E(z) \propto e^{-y|z|}
$$


The effect of a slot is similar to the bulging of the beam pipe. The frequency shift is related to the localization length $L$ of a nivde, $L=1 / 9$,

$$
\frac{\Delta \omega}{\omega}=\frac{q^{2}}{2 h_{c}^{2}}
$$

where $k_{c}=\omega_{c} / c$. Theory ${ }^{[(])}$predicts

$$
q=\frac{k_{c}^{2}}{2} \frac{V_{b}}{S}
$$

where $V_{b}$ is the volume of the bulging.

For an opening, the volume $V_{b}$ should be replaced by the magnetic polarizability $\alpha_{m}$ of the opening. For a long slot in a thick wall, $\alpha_{m}$ is given by Eq. (2). If there are several slots within the localization length $L$, then $\alpha_{m}$ should be multiplied by the number of slots.

For a rectangular beam pipe with dimensions $a \times b, a>b$, and a slot in the upper or lower decks located at $x_{s}, 0<x_{s}<a$

$$
q=a_{m} \frac{(2 \pi)^{2}}{2 a b^{3}} \sin ^{2}\left(\frac{\pi x_{s}}{a}\right)
$$

For a large number of uniformly spaced slots $n_{s}>1, \sin ^{2}(.$.$) can be replaced by 1 / 2$ and $\alpha_{m}$ by the $n_{s} \alpha_{m}$.

The shunt impedance of the trapped mode depends on the resistivity of the wall. For a round beam pipe with radius $b$, the shunt impelance of a trapped $\mathrm{TM}_{0 n}$ mode is ${ }^{(2)}$ :

$$
R_{s}=\frac{Z_{0}}{2 \pi} \frac{\nu}{J_{1}^{2}(\nu)} \frac{b}{\delta}\left(\frac{\alpha_{m}}{\pi b^{3}}\right)^{3}
$$

where $\nu$ is the $n$-th root of the Bessel function $J_{0}(\nu)=0$, and $\delta$ is the skin depth at the frequency $\omega_{c}$. 
The shunt impedance for a rectanguhar chamber with the full dimensions $a \times b$, $a>b$ with a slot in the upper or lower decks is

$$
R_{a}=Z_{0} \frac{a}{b}\left(\frac{1}{k_{c} a}\right)^{5}\left(\frac{b_{e f f}}{\delta}\right)\left[\frac{4 \pi^{2} \alpha_{m}}{b^{3}} \sin ^{2}\left(\frac{\pi x_{a}}{a}\right)\right]^{3} .
$$

Here $k_{c}=\sqrt{(\pi / a)^{2}+(\pi / b)^{2}}, b_{c f f}=a b\left(a^{2}+b^{2}\right) /\left(a^{3}+b^{3}\right)$ is related to the half width of the resonance due to the finite conductivity of the wall

$$
\left(\frac{\Delta \omega}{\omega}\right)_{R W}=\frac{\delta}{b_{e j \delta}}
$$

The theory has been compared and is in good agreement witlt numerical codes ${ }^{[2][3]}$ for a pill-box bulging. We checked the theory using the code MAFIA for slots in a round pipe. Parameters we tsed were: $b=4 \mathrm{~cm}, l=10 \mathrm{~cm}, w=b 0, \theta=12^{\circ}$, number of slots uniformly placed around the boim pipe $n_{s}=18$. The cutoll frequency in this case is $f_{c}=2.8686$ GHz. The theory predicts $\delta_{\omega} / \omega^{\prime}=\tau .22 \times 10^{-3}$ or $f=2.8479$ $\mathrm{GHz}, \Delta f=20.7 \mathrm{MHz}, L=13.8 \mathrm{~cm}$, and $R_{s}=10.1 \mathrm{k} \Omega$. .MAMA finds a mode with $f=2.5 .195 \mathrm{GH} z$ or the frequency shift $\Delta f=f_{c}-f=19.1 \mathrm{MIlz}$ in good agreement wills the theory. The shunt impedance calculated for copper beam pipe and with the magnetic boundary conditions imposed at the distance $z= \pm 75 \mathrm{~cm}$ from the center of the slots was somewhat smaller than in theory, $R_{s}=2.52 \mathrm{k} \Omega$. The Q-factor of the mode is $Q=16000$. The electric loundary conditions at $== \pm 75 \mathrm{~cm}$ give about the same frefuency $f=2.8 .196 \mathrm{GHz}, Q=16620$, and $R_{S}=1.7 \mathrm{k} \Omega$. The field pattern in both cases agrees with exponentially decaying field. The field pat tern confirms also that the magnetic ficdd $H_{\theta}$ leaks into the slot by the length of the order of $w / 2$.

Radiation from a stot may procluce additional width of the resonance and, for a width larger than the frequency sliff, would eliminate the trapped mode. Radiation outside from a slot in a thin wall can give quile noticable width

$$
\left(\frac{\Delta \omega}{\omega \omega}\right)_{\text {rud }}=\frac{\nu^{3} \alpha_{111}^{*} d}{3 b^{5}}
$$

where $\alpha_{m}$ and $q$ are defined by $\mathrm{E}_{\mathrm{l}}$. (2) aud Eq. (\$). Ilowewer, such a radiation for a slot in a thick w'dl $\pi d / w>1$ is suppressed by a factor $c^{-4 \pi l / w^{\prime}} \simeq 2 . \times 10^{-3}$ for PEP.II parameters and is negligibly small. 
Radiation inside the beam pipe for the lowest $\mathrm{TM}_{01}$ mode is possible only into the $T E_{11}$ mode, which frequency is below the cutoff frequency of the $T M_{01}$ mode. The width due to such radiation for a round beam pipe is

$$
\left(\frac{\Delta \omega}{\omega}\right)_{\mathrm{rad}}=\left(\frac{\nu}{2 \mu}\right)^{2}\left(\frac{\alpha_{m}}{\pi b^{3}}\right)^{3} \sqrt{\nu^{2}-\mu^{2}}\left(\frac{\mu^{2}-\nu^{2}}{\mu^{2}}\right) \frac{\sin ^{2}\left(q_{E} l / 2\right)}{\left(q_{E} l / 2\right)^{2}} .
$$

Here $\nu$ and $\mu$ are the roots of the Bessel functions $J_{0}(\nu)=0$ and $d J_{1}(\mu) / d \mu=0$, and $q E=(1 / b) \sqrt{\nu^{2}-\mu^{2}}$ is propagation constant of the TE mode at the frequency of the TM mode. For the lowest $T M_{01}$ mode, $\nu=2.405$ and $\mu=1.88$. However, effect of this radiation is very small. For a symmetric placement of the slots, coupling of the TM mode to the djpole TS mode is suppressed additionally due to azimuthat symmetry.

Conficlence in the theory allows us to analyze the situation in the PEP-II vacuum chamber.

In the straight sections with stainless-steel round beam pipe with radius $b=4.445$ $\mathrm{cm}$ there will be 40 slots with length $l=9 \mathrm{~cm}$ and width $w=3.26 \mathrm{~mm}$. The cutoff frequency $f_{c}=2.5816 \mathrm{GHz}$ is well within the bunch spectrum. The frequency shift of the mode is $\Delta \omega / \omega=3.5 \times 10^{-4}$, which is larger than the width $(\Delta \omega / \omega)=\delta / 2 b=$ $0.36 \times 10^{-4}$ given by the resistivity of the wall. Note, that $\Delta \omega / \omega$ is also larger than the spacing between coupled-mode resonances $\omega_{\text {Tev }} / \omega=5.4 \times 10^{-5}$. The shunt impedance is $R_{s}=85 \Omega$ per pumping port.

In the arcs, there are 18 slots with length $l=15.4 \mathrm{~cm}$ and width $w=2.54 \mathrm{~mm}$ cut in the upper and lower decks of the copper $9 \times 5 \mathrm{~cm}$ rectangular chamber. The cutoff frequency of the lowest TM mode is $f_{c}=3.4296 \mathrm{Gllz}, \Delta \omega / \omega=6 . \times 10^{-4}$. The shunt impedance in the arcs is higher than in the straight sections, $R_{s}=272 \Omega$ per pumping port.

If added together, the shunt impedance of 300 trapped modes in 100 ports in the straight sections and 200 ports in the arcs give the total shunt impedance $R_{s}=$ $62.4 \mathrm{k} \Omega$. Variation of the beam pipe dinensions $\Delta b / b= \pm 12 \mathrm{mil}=0.3 \mathrm{~mm}$ spreads the frequencies of trapped modes around the ring. The frequency spread $\delta \omega / \omega=$ 
$\Delta b / b \simeq 6 \times 10^{-3}$ is large compared to the width of the traped modes $\Delta \omega / \omega=6 \times 10^{-4}$ in the arcs, which give the dominant contribution to the total shunt impedance. Therefore, the maximum total impedance of $N$ pumps, averaged over the variations of the beam pipe dimensions,

$$
Z_{\text {max }}=N R_{s} \frac{\Delta \omega / \omega}{\Delta \omega / \omega+\Delta \omega_{b} / b}
$$

is reduced by a factor of 10 to the exceptable level $Z_{m 4 z}=6.4 \mathrm{M} \Omega$.

The trapped modes may produce two effects. If the frequency of a trapped mode is close (within the width of the resonance $\Delta \omega / \omega=2.4 \times 10^{-4}$ ) to a multiple of the frequency $f_{b}=c / s_{D}$ defined by tlic bunch spacing $s_{B}, f_{b} \simeq 230 \mathrm{MHlz}$, then the resonance may produce aciditional lyeating of the beam pipe. These resonances are spaced by $f_{B}$ and may be controlled by the proper slincension of the beam pipe. The cutoff frequency sloould be away from the multiples of $f_{b}$ and dimensions should be controlled with the accuracy $\Delta b / b<f_{b} / f_{c} \simeq 0.06$ whele $f_{b}$ is the cutoff frequency. Variation of the dimensions of the beant pipe around the ring can bring some of the trapped modes to the resonance. For $I_{b}=3 A$ curren, that would give power $P=I_{b}^{2} R_{s}$ or $P=560 \mathrm{~W}$ per wetum port in the arcs.

The second effect is the resonance with modes of coupled-bunch motion. These resonances are spaced by the revolution frepuency $f_{0}=136 \mathrm{KJlz}$, that is less than the width of a trapped mode and, hence, unavoidable. 'The total inpedinnce of the trapped modes has a probability to give the growth rate larger than the radiation damping time and would require stabilization by the feedback system. That situation can be excluded if we can dosign the pumping ports to avojd trapping.

To destroy the trapped mode we should remember that the localization Eq. (9) and the frecuency shift are defined by the total volume of the bulging. Hence, the trapping does not occur if the beam pipe has a dent with the volume

$$
V_{b}>\alpha_{m}
$$

where $\alpha_{m}$ and $V_{b}$ is the polarizability of all slots and the total volunie of a dent within the localization length of a mode. 
We checked that with MAFIA modifing the model described above by an additional volume protruded inside of the beam pipe, see Fig. 3, with the volume larger than the total polarizability of the slots by $25 \%$. MAFIA finds that the frequency of the mode, which was trapped without the dent, is shifted to the frequency $f=2.8735$ well above the cutoff frequency.

If the beam pipe is recessed to make a smooth bow-like inward bend with the length equal to the length of a slot and with the maximum decrease of the radius $\Delta$ at the center of the slots, it would satisfy the condition $\mathrm{Eq}$. (16) if $\Delta>0.5 \mathrm{~mm}$ in the straight pipes and $\Delta>0.2 \mathrm{~mm}$ in the arcs.

Another way to achieve the same goal is to put a small insertion in the plane transverse to the beam within the localizalion length of the trapped mode. In the arcs, the inserion may have a triangular geomctry with $1 \mathrm{~mm}$ hight at the center of the insertion and width $2.5 \mathrm{~cm}$. To avoid heating by the synchrotron radiation, the insertion may have the $\mathrm{C}$-shape. Such a double slyllow taper produces, of course, some additional broad-band impedance. However, simulations with $\mathrm{ABCI}$ of the impedance of such an insertion in the beam pipe with radius $b=2.5 \mathrm{~cm}$ gives the loss factor $k_{l}=3.7 \times 10^{-4} \mathrm{~V} / \mathrm{pC}$ and inductance $L=7.8 \times 10^{-3} \mathrm{nll}$. For 300 insertions this gives additional inductance $2.4 \mathrm{nH}$ and increase the total machine inductance only by few percent. Two insertions of this kind put at the pumping ports separated by $L=7 \mathrm{~m}$ do not trap a mode between the insertions provided that

$$
\frac{\nu w}{b} \sqrt{\frac{2 \Delta}{b}} \ll 1
$$

For the parameters given above, the width of thew insertion $w=2.5 \mathrm{~cm}$ and the hight, $\Delta=1 \mathrm{~mm}$ this condition is eassly fullfilled both in the straiglat sections $(b=4.5 \mathrm{~cm})$ and the arcs $(b=2.5 \mathrm{~cm})$.

Mode trapping due to imperfections of the beam pipe.

The small recess required to avoid trapping brings in a question about the tolerances on the dimension of the beam pipe. Consider a round beam pipe. A periodic 
variation of the beam pipe radius

$$
b(z)=b_{0}-\Delta \cos (2 \pi z / \lambda)
$$

may result in trapping of a TM HOM with frequency close to the cutoff. A trapped mode has to have a localization length $L$ small compared to the wave length of the perturbation, $L \ll \lambda$, otherwise the average bulging volume would be averaged to zero. The TM mode close to the cutoff freçuency generates a mode trapped in each wave length convolution of the boundary. Each wave length of the perturbation may be considered independently. More accurate consiclcration would show, of course, that the cross-talk of the trapped modes leads to formation of a narrow pass-band but the width of the pass-band, defined by the overlapping of the exponential tails of the indiviclual trapped modes, is small and we can ncglect this effect.

Exact solution of the problem requires consideration of the wave equation with the boundary condition $E_{t}(r=b(z))=0$ or

$$
\left[E_{z}+\frac{d b}{d z} E_{r}\right]_{r=b(z)}=0 .
$$

This is a difficult problem. It can be simplified if we consider only long wave perturbations. In this case, $d b / d z \simeq 2 \pi \Delta / \lambda \ll 1$. Estimating $E_{z}$ from the magnetic flux through the bulging with height $A$,

$$
E_{z} \simeq i\left(\omega_{c} / c\right) \Delta H_{\phi}, \quad H_{\phi} \simeq E_{\mathrm{r}}
$$

we sce that $E_{z}>>U^{\prime} E_{\mathrm{r}}$ provided that $\left.\lambda \gg\right\rangle$. For a trapped mode lacalized at the maximum of the perturbation, $z=0$, with the localizal ion length $L<<\lambda$ the second term in Eq. (18) additionally suppressed by a small factor $2 \pi L / \lambda$ because in this case $d b / d z \simeq\left(\Delta(2 \pi / \lambda)^{2} L\right.$. lience, we can simplify tue boundary condition to $E_{z}(r=$ $b(z)=0$. Then, the solution of the wave equation is $E_{z}(r, z)=J_{0}(\nu r / b(z)) Z(z)$, where $J_{0}(\nu)=0$ and $Z(z)$ satisfies the 1-D wave equation

$$
\left[\frac{d^{2}}{d z^{2}}+\frac{\omega^{2}}{c^{2}}-\left(\frac{\nu}{b(z)}\right)^{2}\right] Z(z)=0
$$

Thus, $(\nu / b(z))^{2}$ plays a tole of the potential barricr. 
This equation can be simplificd for small $\Delta \ll b_{0}$ expanding $b(z)$ over $\Delta$ and can be simplified even further expauding $\cos (2 \pi z / \lambda)$ in $z$ around the minimum $z=0$ for $L<<\lambda$. That gives

$$
\left[\frac{d^{2}}{d z^{2}}+c-q^{4} z^{2}\right] Z(z)=0
$$

where $\epsilon=\frac{\psi^{2}}{\tau^{2}}-\left(\frac{\nu}{b_{0}+\Delta}\right)^{2}$, and

$$
q^{2}=\frac{2 \pi \nu}{\lambda b_{0}} \sqrt{\frac{\Delta}{b_{0}}}
$$

The solution of Eg. (20) is well known. It gives tlie spectrum of the trapped modes $\epsilon_{n}=q^{2}(2 n+1)$. For the lowest mode $n=1$ the solution is Ganssian in $z: Z(z)=$ $Z(0) e^{-q^{2} z^{2} / 2}$. Hence, localization $l=1 / q$, and the assumption we made $L \ll \lambda$ is valid if

$$
\frac{\pi \nu \lambda}{2 b_{0}} \sqrt{\frac{\Delta}{b_{0}}}>>1
$$

Under this condition, there is al. least one 1.rapped mode. 'The maximum number of modes $n_{m a r}$ defined by the condition $(\omega / c)^{2}<\left(\nu /\left(b_{0}-\Delta\right)\right)^{2}$ is

$$
n_{\text {mas }}=\frac{2 \lambda \nu}{\pi b_{0}} \sqrt{\frac{\Delta}{b_{u}}}
$$

Condjition (22) means that $n_{u m a x}>1$.

For the waves of few meters long, Eq. (23) gives very small $\Delta$. Consider, for example, the modulation with $\lambda=3 \mathrm{~m}$ in the beam pipe $h_{0}=3 \mathrm{~cm}$. The trapped mode exist if $\Delta>0.2 \mu m$.

The width of the mode $\delta \omega / \omega$ is smaller than the width due to resistive wall if

$$
\frac{\pi \nu \lambda}{l_{11}} \sqrt{\frac{\Delta}{b_{0}}}>\frac{\delta}{b_{0}}\left(\frac{\nu \lambda}{2 b}\right)^{2}
$$

This condition is stronger that $\mathrm{E}_{\mathrm{l}}$. (23) for $\lambda>(2 b / \nu) \sqrt{b / \delta}$. For thic arcs that means $\lambda>8 \mathrm{~m}$. 
The shunt impedance of a trapped mode is given by Eq. (11) where $\alpha_{m}$ has to be replaced by the volume $V_{b}=2 \pi b_{0} \Delta / q$ with q defined in Eq.(21):

$$
R_{s}=\frac{Z_{0}}{2 \pi} \frac{\nu}{J_{1}^{2}(\nu)} \frac{b}{\delta}\left(\frac{2 \lambda}{\pi \nu b}\right)^{3 / 2}\left(\frac{\Delta}{b}\right)^{y / 4}
$$

or

$$
R_{s}=78.7 \frac{b}{\delta}\left(\frac{\lambda}{b}\right)^{3 / 2}\left(\frac{\Delta}{b}\right)^{9 / 4} \Omega
$$

The shunt impedance decreases rapidly with the amplitude $\Delta$. However, it is quite noticable even for small amplitudes for long wave length. Take $\Delta=10 \mu m, \lambda=3 \mathrm{~m}$, $b=3 \mathrm{~cm}$. Then $R_{a} \simeq 400 \Omega$.

The question remains if there is a limit on small $\Delta-s$ which still can trapped mode. One of such a meclianism is the random variations of the radius $b(z)=b_{0}+\delta_{\mathrm{r}}(z)$. Let $\delta(z)$ be a random noise, $\left\langle\delta_{\mathrm{r}}\right\rangle=0,\left\langle\delta_{r}^{2}\right\rangle=\delta_{0}^{2}$, with the correaltion length $\zeta$.

Effect of the noise can be explained considering probability of the trapped mode to leak under the bartier of Eq. (19) to the nciglbouring potential well. Large probability of the leak means low external $Q$-factor and damping of the mode- Normally, probability of the tunneling is exponentially small, $P \propto e^{-q \lambda}$ because $q \lambda=\lambda / L>>1$. Replace, however, the potential barrier with the length $\lambda$ by $n$ small barriers each with the same hight but with the length $\lambda / n$ separated by small gaps of the same width. Penetration through a single sinall barrier cin be deacribed by the matrix relation

$$
\left(\begin{array}{l}
\psi_{1} \\
\psi_{2}
\end{array}\right)=S\left(\begin{array}{l}
\psi_{3} \\
\psi_{4}
\end{array}\right) ; \quad S=\left(\begin{array}{cc}
1-\epsilon & \epsilon \\
-\epsilon & 1+\epsilon
\end{array}\right)
$$

where $\epsilon=e^{2 q \lambda / n}-1$, and $\psi_{1}, \psi_{2}$ are amplitudes of the waves jncoming and reflected from the barriel from the left, and $\psi_{4}, \psi_{3}$ are the amplitudes of the waves incoming and reflected from the barrier respectively. We assumc, that the gaps are narrow and phase advance per barrier is very small. In this approximation, all $\psi$-s are real. 
Multiplying the matrices, we got

$$
S=\left(\begin{array}{cc}
1-n \epsilon & n \epsilon \\
-n \epsilon & 1+n \epsilon
\end{array}\right)
$$

The probality io penetrate through in small barricts can be obtained from that imposing condition $\psi_{4}=0$. That gives

$$
\psi_{1}=\frac{1}{1+n \epsilon} \psi_{1}
$$

For $n=1$, ancl $g \lambda>i$ that gives exponemtially small probals.ity, fut the limit $n \rightarrow \infty$, $q \lambda \gg 1$, the probability to petuetiate throtigh the total barrier is $P=(1+2 q \lambda)^{-2}$ rather than being exponentially mall.

This example shows, that if the amplitude of a noisc of the boundary is comparable with the amplitude of the periodic wave the penetration through the barrier is much larger than for the exponentially small tunneling under the barrier. The result corresponds to diffusion of the wave through small barriers and, therefore, should be valid not only for equal but also for large number of small but otherwise arbitrary barriers including random noi:ic. In particular, that means that random perturbation of the boundary can not produce trapped modes.

\section{Summary}

The vacuum ports will be screend with slots. It is shown that slots do not screen pumps from the TE HOM-s propagating in lle beam pite.

Mode trapping produced by the leak of the magnetic ficld in the slots may cause mode trapping. 'This effect, prexlicted theoretically, is confirned by MAFIA and in a good agreement with theory. The width of these modes given by resistive wall and radiation is found to be stmall. Wie discuss the menns to avoid these modes, which are potentially clangerous for henting ol the bean pipe and the longitudisal coupled-buch iustability.

One of us (S.11.) wants to thanks F. Ilenestroza, K. Ko, and $\mathrm{C}$. Ng for their guidance and help with MAPL simulations. We appreciate comments by J. Seernan and G. Lambertson. 


\section{Figure Captions}

1. Fig. 1 Dependence of $E_{z}$ of the trapped mude on the distance from the slot center $(z=0)$.

2. Fig. 2 Leak of the magnetic field of a trapped mode into the slot.

3. The same as in Fig. 2 but with insertions. The frequency of the lowest mode is pushed above cutoff.

\section{REFERENCES}

1. S. S. Kurennoy Beam Coupling Impedance of Iloles in Vacuum Chamber Walls, IHEP 92-84, UNK, 1992

2. G. Stupakov, S. Kurennoy Trapped Electromagnetic Modes in a Waveguide with a Small Discontinuity, SSCL-Preptint-159, June 1993

3. C.K. Ng, T. Weiland, Impedance Analysis of the PEP-II Vacuum Chamber, PEP.II Note, 1994 


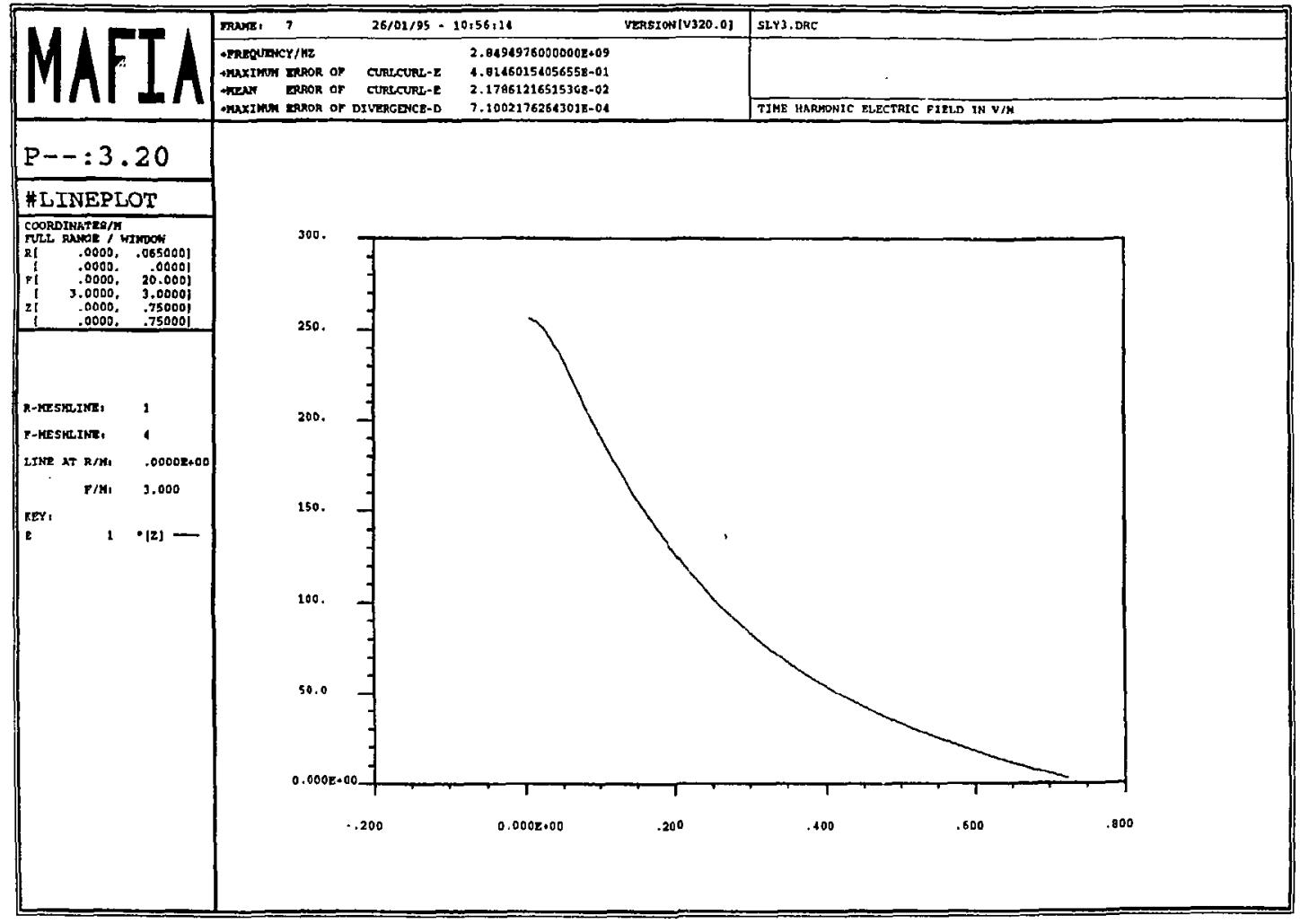




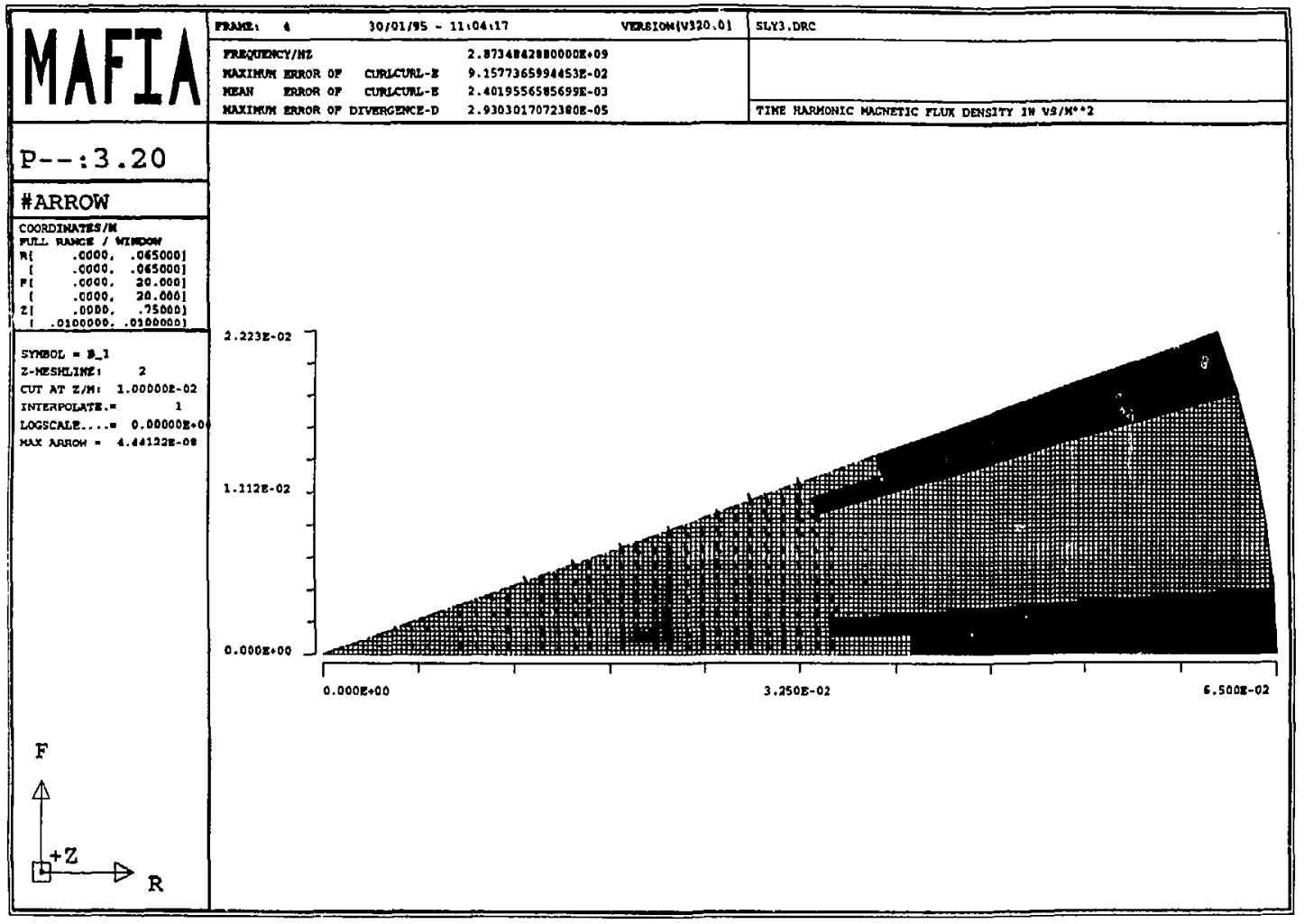




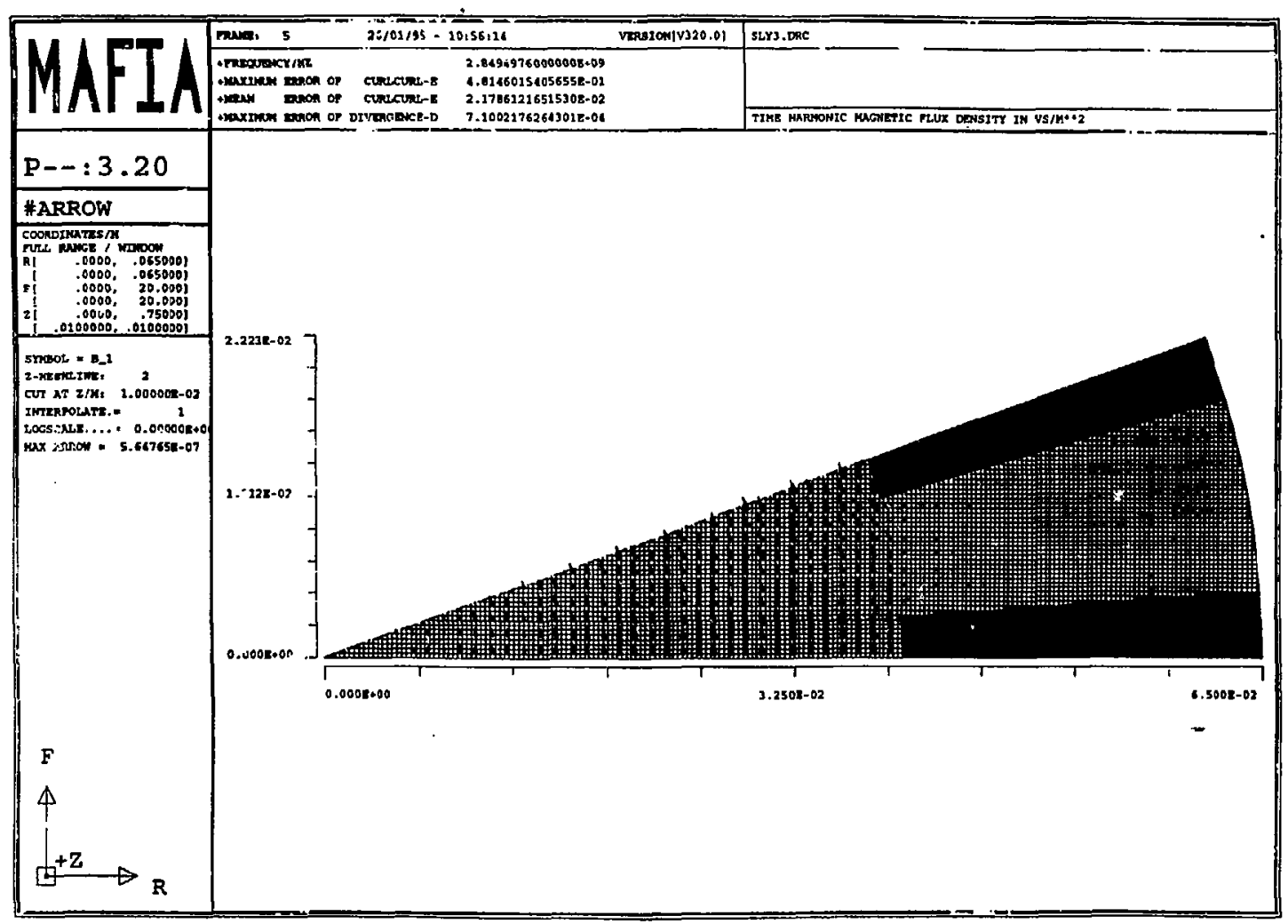

